\title{
CHANGING TRENDS IN CHRONIC PANCREATITIS
}

Sreenidhi G.M ${ }^{1}$, Nikshita $\mathrm{N}^{2}$

\section{HOW TO CITE THIS ARTICLE:}

Sreenidhi G. M, Nikshita N. "Changing Trends in Chronic Pancreatitis". Journal of Evolution of Medical and Dental Sciences 2014; Vol. 3, Issue 08, February 24; Page: 1864-1871, DOI: 10.14260/jemds/2014/2071

ABSTRACT: BACKGROUND: AIMS: To determine the demographic profile, to evaluate risk factors of chronic pancreatitis, frequency of complications and therapeutic modalities for management of chronic pancreatitis. METHODS: Data analyzed retrospectively from 177 patients of chronic pancreatitis admitted in the Department of Surgery in our institute between Jan 2003 \& December 2012. RESULTS: Male predominance, mean age of presentation is 32yrs, $66 \%$ with Alcohol consumption was the main risk factor, with associated diabetes and gall stones. Pain abdomen was the commonest mode of presentation, and USG sensitivity rate was 55\%. Parenchymal calcification, Ductal calculi, pseudocyst were the commonest complications. Medical line of management was the initial therapy and surgical intervention was done as indicated. Number of readmissions noted. CONCLUSIONS: Although Kerala is known for highest prevalence of chronic pancreatitis in our country, it is a noted disease \& is on the rise in the state of Karnataka. Mean age of onset is older as compared to two decades ago and also a shift of etiology from tropical to alcoholic pancreatitis has been noted.

KEYWORDS: Chronic pancreatitis, Tropical pancreatitis, recurrent acute pancreatitis

INTRODUCTION: Chronic pancreatitis is a progressive inflammatory and fibrotic disease of the pancreas with hallmark features of abdominal pain, malabsorption, malnutrition, diabetes mellitus, and pancreatic calcifications. There is irreversible progressive destruction of pancreatic tissue. It is a heterogeneous disease, multifactorial, variable in presentation with Geographic variation.

The prevalence of tropical pancreatitis is estimated to be 126/100, 000 population in southern India. ${ }^{1}$ This is in contrast to the estimated prevalence of chronic pancreatitis of around 10-15/100, 000 population in several western industrialized countries and 45.4/100, 000 population in Japan.2, ${ }^{3}$ Such a high prevalence of chronic pancreatitis in India suggests that it is an endemic zone for chronic pancreatitis and points towards a possible genetic and/or environmental factor as playing an important etiologic role.

The spectrum of chronic pancreatitis in India is variable. There are a number of studies on profile of chronic pancreatitis in south India. ${ }^{1,4-6}$ The clinical spectrum of chronic calcific pancreatitis has been found to be different in northern India. ${ }^{7}$ Heavy consumption of cassava has been implicated in the etiopathogenesis of tropical pancreatitis in Kerala. Regarding tropical pancreatitis, several hypotheses have been proposed, in particular, the malnutrition theory, the cassava hypothesis and the oxidant stress hypothesis. ${ }^{8}$ The largest series has however, been described from South India by Geevarghese. ${ }^{9}$ The clinical profile, complications and response to therapy may be different in different parts of India.

It has become accepted that alcohol-induced Recurrent acute pancreatitis evolves into chronic pancreatitis, 10 characterized by a sequence of necrotic and fibrotic events, originally proposed in 1946 by Comfort et al. ${ }^{11}$ Some patients may have subclinical chronic pancreatitis from the onset of the disease, which could explain why chronic pancreatitis is present in $40 \%$ with the 
first attack of pancreatitis ${ }^{12}$ and in $10 \%$ of chronic heavy alcohol consumers (defined by consuming at least $80 \mathrm{~g} /$ day of alcohol for 5 years or longer) without prior attacks of pancreatitis. ${ }^{13}$

Three new studies ${ }^{14-16}$ clarify further the association of smoking and drinking alcohol with chronic pancreatitis, acute pancreatitis, and/or recurrent acute pancreatitis (RAP).

In our study, the increasing number of cases to be diagnosed with chronic pancreatitis with alcohol consumption as a predominant etiological factor from Karnataka has been shown, as the largest prevalence of cases in literature have been reported from Kerala mainly due to cassava consumption as an etiological factor.

AIMS AND OBJECTIVES: To determine the demographic profile, to evaluate risk factors of chronic pancreatitis, frequency of complications and therapeutic modalities for management of chronic pancreatitis.

MATERIAL AND METHODS: This is a retrospective study done at our institute between January 2003 to December 2012 and a total number of 177 cases were included in the study.

INCLUSION CRITERIA: All patients with chronic pancreatitis, sonologically confirmed.

Data collection included a detailed history and clinical examination findings and findings of routine blood, serum amylase and lipase, radiological investigations included ultrasound, chest xray, and contrast enhanced CT abdomen, management and the number of readmissions. Data was entered in the proforma, tabulated and analyzed.

RESULTS: 177 cases of chronic pancreatitis admitted at our institute from Jan 2003 to Dec 2012 included in the study. Various parameters to analyze data included, age and sex incidence, mode and time of presentation, ultrasound and CT findings, complications and management (medical/surgical) protocol and number of readmissions were taken into account.

1. Year wise distribution of cases: The number of cases of chronic pancreatitis has increased over the years (figure 1).

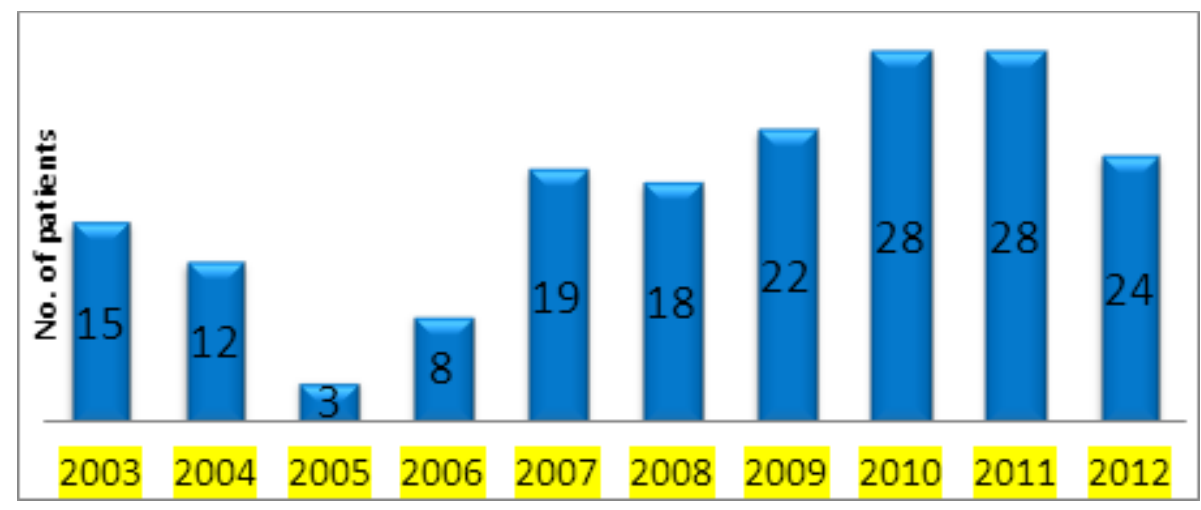

Figure 1 


\section{ORIGINAL ARTICLE}

2. Age and Sex distribution: The age distribution of the 177 cases of chronic pancreatitis ranged from 7years to 72years and had maximum number of patients in the 31-40yrs age group. This study had 25 female and 152 male patients, female to male ratio being 1: 4.3(figure 2).

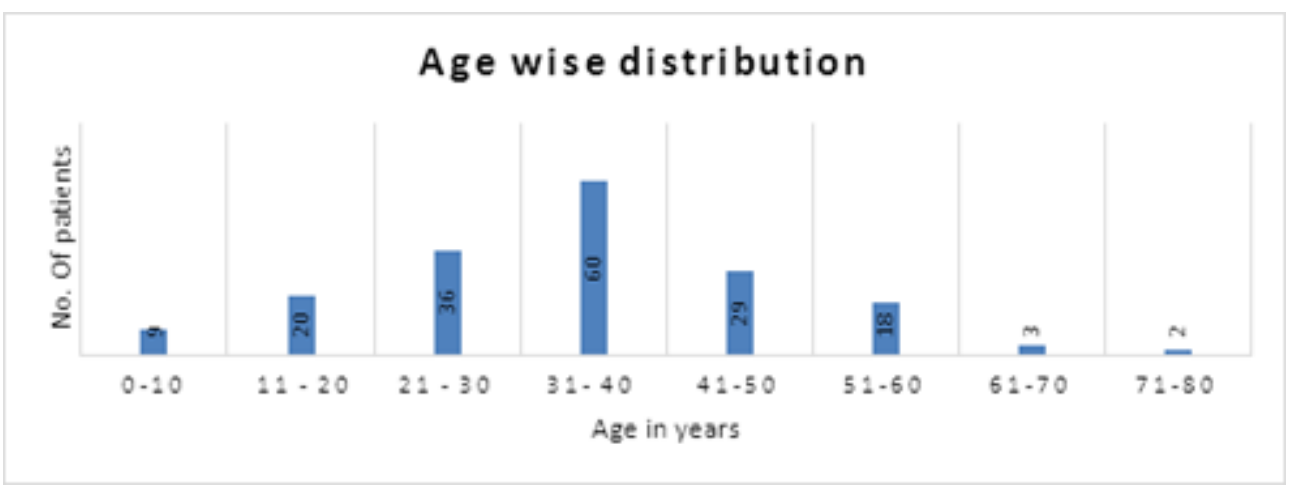

\section{Figure 2}

3. Mode of presentation: Of 177 patients, $99 \%$ cases presented with history of pain abdomen, $54.2 \%$ cases with vomiting, $11.2 \%$ had steatorrhea, jaundice in $8.4 \%$ cases and fever in $14.1 \%$ cases (figure 3).

\begin{tabular}{|l|c|c|}
\hline \multicolumn{1}{|c|}{ Symptoms } & Number of patients & Percentage \% \\
\hline Pain abdomen & 175 & 99 \\
\hline Vomiting & 95 & 54.2 \\
\hline Fever & 25 & 14.1 \\
\hline Steatorrhea & 20 & 11.2 \\
\hline Jaundice & 15 & 8.4 \\
\hline \multicolumn{3}{|c|}{ Figure 3 } \\
\hline
\end{tabular}

4. Risk factors: $66 \%$ cases were alcoholic, $14 \%$ had history of smoking, $1.7 \%$ has family history of chronic pancreatitis, and idiopathic in $14 \%$ cases. (Figure 4 ).

\begin{tabular}{|l|c|}
\hline \multicolumn{1}{|c|}{ Risk factors } & Percentage \% \\
\hline Alcohol consumption & 66 \\
\hline Smoking & 14 \\
\hline Family history & 1.7 \\
\hline Idiopathic & 14 \\
\hline \multicolumn{2}{|c|}{ Figure 4 } \\
\hline
\end{tabular}

5. Associated findings: Out of 177 patients, 72 patients were diabetic, 11 patients had calculous cholecystitis, 5 cases had choledocholithiasis and 4 cases had cirrhosis of liver.(figure 5) 


\begin{tabular}{|l|c|}
\hline \multicolumn{1}{|c|}{ Associated findings } & No. of patients \\
\hline Diabetes mellitus & 72 \\
\hline CBD calculi & 5 \\
\hline Calculous Cholecystitis & 11 \\
\hline Cirrhosis of Liver & 4 \\
\hline \multicolumn{2}{|c|}{ Figure 5 } \\
\hline
\end{tabular}

\section{Investigations:}

a. Serum and Serum Lipase: Serum amylase was routinely sent in all cases, and serum lipase in few cases which had elevated serum amylase and in patients with features of acute exacerbation of chronic pancreatitis. (Figure 6).

b. Ultrasound and CT findings: The commonest findings on CT Abdomen were pancreatic parenchymal calcification, ductal calculi, pseudocyst, which in many cases was not detected on ultrasound imaging. The sensitivity of Ultrasound in the diagnosis of complications of chronic pancreatitis in this study was 55\%.

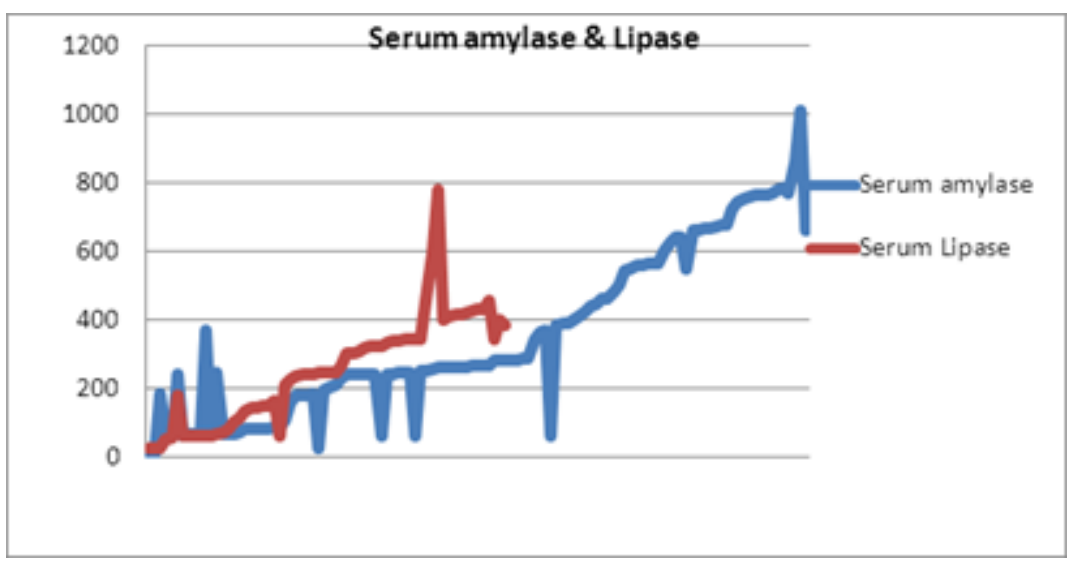

Figure 6

7. Complications of Chronic Pancreatitis: $79 \%$ of patients had associated complications of chronic pancreatitis which included Parenchymal calcification (52\%), pancreatic ductal calculi (26\%), pancreatic pseudocyst (27\%), portal vein thrombosis (4.5\%), ascites (2.8\%). Out of these cases, $26 \%$ had all three complications simultaneously - calcification, ductal calculi and pseudocyst.

8. Management protocol: 83 cases underwent medical line of management, while 94 cases were advised surgical intervention.

Medical line of treatment was the initial therapy, with control of diabetes, symptomatic treatment with rehydration, analgesics, antiemetics, antacids, antibiotics when indicated, correction of electrolyte imbalance, enzymes, somatostatin analogues (octreotice), calcium gluconate and nutritional support. 
Surgical Intervention was advised in 94 cases, out of which 20 cases were lost on follow up and 74 cases underwent surgical intervention. (Figure 7)

The protocol for indications for surgery in chronic pancreatitis cases in our institute included:

Longitudinal pancreaticojejunostomy - intractable pain, not relieved after initial medical line of management, along with dilated pancreatic ducts $>5 \mathrm{~mm}$, ductal calculi, multiple duct strictures with parenchymal calcification.

Frey's procedure - pathology was confined to head of pancreas

Distal pancreatectomy - ductal calculi/calcification confined to tail of pancreas.

Whipple's procedure, in our study was done in a patient with annular pancreas. Various procedures were done for pancreatic pseudocyst.

Endotherapy which included ERCP along with sphincterotomy and extraction of CBD calculi (4 cases) with CBD stenting was done, and endotherapy is being done more frequently since recent years.

Cholecystectomy was done along with LPJ in 3 cases and Laparoscopic cholecystectomy in 7 cases and in 4 cases it was done following ERCP.

\begin{tabular}{|c|c|c|}
\hline \multicolumn{2}{|c|}{$\begin{array}{r}\text { Surgical procedures } \\
\end{array}$} & No. of patients \\
\hline \multicolumn{2}{|c|}{ Longitudinal Pancreatico-jejunostomy } & 27 \\
\hline \multicolumn{2}{|c|}{ Frey's procedure } & 15 \\
\hline \multirow[t]{5}{*}{ Procedures for Pseudocyst } & Cystogastrostomy & 4 \\
\hline & Cystojejunostomy & 3 \\
\hline & CT/USG guided aspiration & 3 \\
\hline & Laparomtomy+External drainage & 1 \\
\hline & Laparoscopic Cystogastrostomy & 3 \\
\hline \multicolumn{2}{|l|}{ Distal Pancreatectomy } & 3 \\
\hline \multicolumn{2}{|l|}{ Whipple's procedure } & 1 \\
\hline \multicolumn{2}{|l|}{ Cholecystectomy + LPJ } & 3 \\
\hline \multicolumn{2}{|c|}{ Laparoscopic Cholecystectomy } & 7 \\
\hline
\end{tabular}

9. Readmissions: Out of 83 cases medically managed cases, 10 cases were readmitted for pain thrice in a year, 18 cases twice a year, and 25 cases once in a year.

In 70 cases managed surgically, 4patients readmitted post LPJ, 2 readmissions post Frey's procedure and 3 readmissions post procedures for Pseudocyst. These patients were given symptomatic support.

DISCUSSION: In our study, 177 cases of chronic pancreatitis were included. We noted that the average age of presentation was 32 years, with a male to female ratio being 4.3:1. Alcohol consumption was the most significant risk factor. Diabetes mellitus and calculous cholecystitis were other associated findings. The commonest complications observed were parenchymal calcification, pancreatic ductal calculi and pseudocyst. Patients underwent initial medical line of therapy and surgical intervention when indicated. The number of readmissions was noted as patients with chronic pancreatitis commonly present with recurrent bouts of pain. 
It is often difficult to differentiate recurrent acute pancreatitis from exacerbations of chronic pancreatitis. Even today, in certain situations, the correct diagnosis can often be achieved only on follow up of the patient. ${ }^{17}$ In all our patients the diagnosis of chronic pancreatitis was confirmed by imaging studies.

With better understanding of the illness, it seems that the natural history of Tropical chronic pancreatitis previously described by Geevarghese ${ }^{9}$ as pain between the ages of 6-10 years, diabetes by the age of 20 years and death before age of 30 years has definitely changed.

In a prospective nationwide study in India ${ }^{18}$, the authors found that the majority of patients had pancreatitis of unknown origin ( $60 \%$ of the cases); alcoholic chronic pancreatitis accounted for a third of the cases, whereas tropical pancreatitis was present in only 3.8\% of the cases. It seems that alcohol tends to be increasing in frequency in India, as is chronic pancreatitis of unknown etiology.

Changing lifestyle may contribute to the changing frequencies of the various etiologies of chronic pancreatitis. This is the reason why, from 2004 to the present, the etiological features of chronic pancreatitis have been reported to be different compared to those in the past. For example, in Korea ${ }^{19}$, the main etiological factor remains alcohol $(64.3 \%)$ followed by an unknown etiology (20.8\%), obstruction (8.6\%) and autoimmune pancreatitis $(2.0 \%)$. In a recent survey on chronic pancreatitis in the Asian-Pacific region ${ }^{20}$, there was a great variability in the frequency of alcoholic pancreatitis, accounting for about $19 \%$ of chronic pancreatitis cases in China to $95 \%$ in Australia,

The impact of changing lifestyle, especially in developing countries, may contribute to modifying the etiology of chronic pancreatitis. For example, alcohol consumption in developing countries may increase ${ }^{21}$ and this could change the etiology of chronic pancreatitis in these countries.

CONCLUSION: There is a rise in the disease pattern of chronic pancreatitis in the state of Karnataka too, while our neighboring state, Kerala is known for highest prevalence of Chronic Pancreatitis in the world, which can be attributed to changing lifestyle leading to a shift in etiology from tropical to Alcoholic pancreatitis, and an older age of onset of disease. Management protocol can achieve optimized results in these patients in treating chronic pancreatitis patients.

\section{REFERENCES:}

1. Balaji LN, Tandon RK, Tandon BN, Banks PA. Prevalence and clinical features of chronic pancreatitis in southern India. Int J Pancreatol 1994; 15: 29-34.

2. The Copenhagen pancreatic study group: An interim report from a prospective epidemiological multicentre study. Scand J Gastroenterol 1981; 16:305-312.

3. Lin Y, Tamakoshi A, Matsuno S, Takeda K, Hayakawa T, Kitagawa M, Naruse S, Kawamura T, Wakai K, Aoki R, Kojima M, Ohno Y, Nationwide epidemiological survey of chronic pancreatitis in Japan . J Gastroenterol 2000; 35:136-141.

4. Chari ST, Mohan V, Jayanthi V, Snehalatha C, Malathi S, Viswanathan M, Madanagopalan N. Comparative study of the clinical profiles of alcoholic chronic pancreatitis and tropical chronic pancreatitis in Tamil Nadu, south India. Pancreas 1992; 7: 52-8.

5. Balakrishnan V, Sauniere JF, Hariharan M, Sarles H. Diet, pancreatic function, and chronic pancreatitis in south India and France. Pancreas 1988; 3:30-5. 
6. Mori M, Hariharan M, Anandakumar M, Tsutsumi M, Ishikawa O, Konishi Y, Chellam VG, John M, Praseeda I, Priya R, Narendranathan M. A case-control study on risk factors for pancreatic diseases in Kerala, India. Hepatogastroenterology 1999; 46:25-30.

7. Rai RR, Acharya SK, Nundy S, Vashisht S, Tandon RK. Chronic calcific pancreatitis: profile in northern India. Gastroenterol Jpn 1988; 23: 195-200.

8. Martin E, Bedossa P. Diffuse fibrosis of the pancreas: a peculiar pattern of pancreatitis in alcoholic cirrhosis. Gastroenterol Clin Biol. 1989; 13:579-584.

9. Geeverghese PH. Pancreatic diabetes; A clinicopathological study of growth onset diabetes with pancreatic calculi, Bombay: Popular Prakastan, 1968.

10. Ammann RW. Alcoholic chronic pancreatitis: its relation to alcoholic acute pancreatitis. Gastroenterol Clin Biol 1996; 20:312-314.

11. Comfort MW, Gambill EE, Baggenstoss AH. Chronic relapsing pancreatitis: a study of 29 cases without associated disease of the biliary or gastro-intestinal tract. Gastroenterology 1946; 6:376-408.

12. Ammann RW, Buehler H, Bruehlmann W, et al. Acute (nonprogressive) alcoholic pancreatitis: prospective longitudinal study of 144 patients with recurrent alcoholic pancreatitis. Pancreas 1986; 1:195-203

13. Ammann RW, Muellhaupt B, Meyenberger C, Heitz PU. Alcoholic nonprogressive chronic pancreatitis: prospective long-term study of a large cohort with alcoholic acute pancreatitis (1976-1992). Pancreas 1994; 9:365-373

14. Tolstrup JS, Kristiansen L, Becker U, Gronbaek M. Smoking and risk of acute and chronic pancreatitis among women and men: a population-based cohort study. Arch Intern Med 2009; 169:603-609.

15. Yadav D, Hawes RH, Brand RE, et al. Alcohol consumption, cigarette smoking, and the risk of recurrent acute and chronic pancreatitis. Arch Intern Med 2009; 169:1035-1045

16. Irving HM, Samokhvalov AV, Rehm J. Alcohol as a risk factor for pancreatitis. A systematic review and meta-analysis. JOP 2009; 10:387-392.

17. Talamini G, Falconi M, Bassi C, Mastromauro M, Salvia R. Chronic Pancreatitis: Relation to Acute Pancreatitis and Cancer. J Pancreas 2000; 1(Suppl.): 69-76.

18. Balakrishnan V, Unnikrishnan AG, Thomas V, Choudhuri G, Veeraraju P, Singh SP, Garg P, Pai CG, Devi RN, Bhasin D, et al. Chronic pancreatitis. A prospective nationwide study of 1, 086 subjects from India. JOP. 2008;9:593-600

19. Ryu JK, Lee JK, Kim YT, Lee DK, Seo DW, Lee KT, Kim HG, Kim JS, Lee HS, Kim TN, et al. Clinical features of chronic pancreatitis in Korea: a multicenter nationwide study. Digestion. 2005; 72:207-211.

20. Garg PK, Tandon RK. Survey on chronic pancreatitis in the Asia-Pacific region. J Gastroenterol Hepatol. 2004;19:998-1004.

21. Das SK, Balakrishnan V, Vasudevan DM. Alcohol: its health and social impact in India. Natl Med J India. 2006; 19: 94-99. 


\section{ORIGINAL ARTICLE}

\section{AUTHORS:}

1. Sreenidhi G. M.

2. Nikshita N.

\section{PARTICULARS OF CONTRIBUTORS:}

1. Associate Professor, Department of General Surgery, Kempegowda Institute of Medical Sciences Hospital \& Research Center.

2. Post Graduate, Department of General Surgery, Kempegowda Institute of Medical Sciences Hospital \& Research Center.

\section{NAME ADDRESS EMAIL ID OF THE}

\section{CORRESPONDING AUTHOR:}

Dr. Sreenidhi G.M,

Kempegowda Institute of Medical Sciences Hospital \& Research Center,

K. R. Road, V. V. Puram, Bangalore.

E-mail: nidhikavitha@yahoo.com

Date of Submission: 04/02/2014.

Date of Peer Review: 05/02/2014.

Date of Acceptance: 13/02/2014.

Date of Publishing: 19/02/2014. 